\title{
DAC-EuCheMS Lecture Award presented to ABC author Freddy Adams
}

\section{Nicola Oberbeckmann-Winter ${ }^{1}$ (D)}

Published online: 14 January 2020

(C) Springer-Verlag GmbH Germany, part of Springer Nature 2020

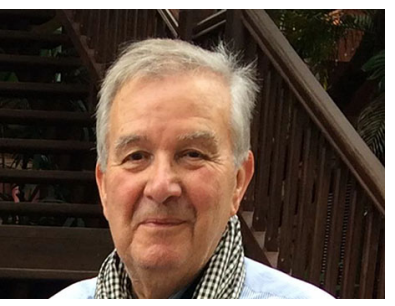

Freddy Adams is Emeritus Professor at the University of Antwerp, Belgium, with teaching assignments in analytical chemistry and research in analytical chemistry and its applications in the materials sciences, the environment sciences and art \& archaeology. His present research interests concern general aspects of analytical chemistry and chemical imaging, particularly with X-ray methods and ultra-trace analysis with mass spectrometry. He was co-founder and Director of the Micro-and Trace Analysis Centre (MiTAC, 1980-2003) and Rector of the Graduate School of the UA (1983-1995). He was member of the Flemish Interuniversity Council (1983-1995 and chairman of the Council 1988-1990, member of the Belgian National Research Council (1989-1995), the Flemish Council for Scientific Research (1994-1998), the Flemish Industrial Research Board (1995-1997) and Vice-President of the Flemish Institute for Biotechnology (1995-2004). He was a member of the IUPAC Analytical Division Committee (1998-2002), member of the Steering Committee of Science for Peace Program of NATO (19972004), President-General of the Royal Flemish Chemical Society (1995-1998) and member of the Scientific Advisory Committee of the European Synchrotron Radiation Facility (ESRF, Grenoble, 1989-2000). $\mathrm{He}$ is author or co-author of five books and over 500 publications in international scientific journals.

Honours and awards include the Louis Gordon Memorial Award, 1979; Castaing Award, 1984; Environment Policy Prize of the Coca-Cola Foundation, 1990; Pregl Medal of Austrian Chemical Society, 1996; Doctor Honoris Causa, University of Iasi, Romania, 1998; "Torch" Award of the Winter Plasma Conference on Plasma Spectrochemistry,

Nicola Oberbeckmann-Winter

nicola.oberbeckmann-winter@ springer.com

1 Analytical and Bioanalytical Chemistry, Springer Verlag, Tiergartenstrasse 17, 69121 Heidelberg, Germany
Norway, 2001; and Doctor Honoris Causa University of Chisinau, Moldova, 2005.

The DAC-EuCheMS Lecture Award, sponsored by Springer-Verlag, recognizes a European individual who has demonstrated significant and sustained achievements in research or education in analytical chemistry throughout his or her career. Freddy Adams is the recipient of the 2019 DAC-EuCheMS Award. His lecture, entitled "The Metamorphosis of Analytical Chemistry: chemical analysis in the 21 st century", was presented at Euroanalysis XX held in Istanbul, Turkey, from September 1 to $5,2019$.

Freddy Adams talked to Nicola Oberbeckmann-Winter about his research, his motivation, and himself.

\section{What motivated you to become a researcher and what do you find most rewarding in your professional life?}

I grew up in the post-Second World War period that was dominated by the expected blessings of abundant nuclear energy, the hesitant advent of solid-state electronics and telecommunication and, on the other side, the frightening Cold War and the potential nuclear holocaust, MAD - mutually assured destruction. As a teenager, my interest in science was fed by inspiring high school teachers of physics and chemistry; for science and technology these were the Roaring Sixties. This led me to select chemistry at the university. The start of a $\mathrm{PhD}$ and life as a researcher came quite naturally; I was offered a position without any solicitation, as research associate of the Belgian Interuniversity Institute of Nuclear Sciences and stationed at Ghent University. My application for a professorship at the graduate school of the University of Antwerp, a newly founded university, a product of the university expansion in Belgium, was the only application for work of my entire life. Young scientists today have quite different, less predictable career prospects. 
Curiosity-driven research work was my ambition from early on in my life. The most rewarding aspect of my career was the freedom I enjoyed, together with the pleasure of working in the intellectually inspiring environment of a young and dynamic university. I considered - and still consideranalytical chemistry to be an excellent discipline to work in. It belongs to "Pasteur's quadrant" of science, use-inspired basic research, as opposed to pure basic research, exemplified by the work of Niels Bohr (Bohr's quadrant), or the pure applied research of the Edison quadrant. I quote here the popular book by Donald Stokes, Pasteur's Quadrant: Basic Science and Technological Innovation, 1997.

\section{From where did you start and how did your most recent work relate to/differ from your scientific roots?}

Sensitive instrumental analysis was my main interest, established from my early experiences. In the early $1960 \mathrm{~s}$ there was much less to choose from than today, for nondestructive analysis, arc and spark emission spectroscopy, some X-ray fluorescence and, as a cherry on the cake, neutron activation analysis with the small local research reactor at Ghent University, used with gamma-ray spectrometry for measurement. Moving to Antwerp I shifted my research to inorganic spark source mass spectrometry and energy-dispersive $\mathrm{X}$-ray analysis, and from there-on the wave of the turbulent development of instrumental analysis - to microscopic elemental analysis and imaging, speciation and molecular analysis with mass spectrometry and laboratory X-ray sources and, moving back to big instrumentation, the new European synchrotron radiation source in Grenoble.

The basic principles of the later work in my career did not differ so much from those in my early years. Changes did not affect reality on a deeper level than to cement the status quo. It is only near the end of my professional life that real changes started appearing with the advent of the Big Data Era, the Information Age, in the early years of the twenty-first century. Analytical chemistry and various applications in chemical analysis started to participate in the gathering of huge volumes of information with high speed and with minimum labor and cost, capturing a variety of structured and unstructured information together with other salient information of the object studied. The study object changed too: from homogeneous to heterogeneous, from ordered to disordered, from simple to complicated. This revolutionary, instead of evolutionary, change and its profound implications for the discipline are the subject of the paper accompanying this interview. Some analytical chemists might react and not recognize the ongoing paradigm shift, a situation not unlike the attitude of some against the now-crying evidence of global change. Changes that are perceived as too fast and too radical seem to induce conservative, sometimes irrational reactions.

\section{Who inspired you most during your career?}

Lasting inspiration comes early on in the development process, I mentioned already my high school teachers in the physical sciences. Early in my scientific development my prime and foremost inspiration came from the late professor Julien Hoste (1921-2012) at Ghent University during my PhD and my postdoc period in his laboratory. He transmitted knowledge, wisdom, even culture, while simultaneously he left me freedom of opinion and thought. If I could express anything apologetic in this talk, it would be that I have missed the opportunity to tell him how important he was in the formative years of my life as a scientist in his final years; he passed away in 2012.

\section{What is the trickiest problem you have had to overcome in that research? How did you solve it?}

The constant worry of securing investment for up to date analytical instrumentation ... and the surprise that, in the end, it was possible to get everything that was really needed, instrumentation and human capital, for keeping on track for the research we wanted to do. It is with time that I realize that this is not providence but the result of a healthy and diversified research funding system with a range of funding agencies.

\section{Where do you consider your field is heading?}

In the Information Age, present day society is getting smarter and more complex. Cross-disciplinary and trans-disciplinary approaches have started dominating the scene, and this is observed across the entire scientific world. Analytical chemistry is developing as a major player in all this. We have access now to an extensive, quasi-unlimited range of powerful analytical instruments; given the creative imagination of analytical scientists, anything imaginable is possible: single atom or molecule detection, spatial resolution at or near the atomic level, unprecedented speed and ease of analysis, etc. Analytical instruments, together with many other new observational tools, can be used for data-intensive exploration, opening up a range of new ways to make sense of nature and providing new explanations of phenomena and processes.

Let me make this clear with the most challenging research concerning the visualization and determination of biologically 
relevant molecules in molecular cell biology. Chemical analysis is an important component in all this and is confronted with several challenges. In the first place, subcellular structures and molecular complexes essential for biological function exist on length scales from the micrometer to nanometer, hence their analysis requires a spatial resolution considerably below the optical diffraction limit. Secondly, there is a need for extremely fast analysis to match the existing omics tools. The rapid development of 3D cellular imaging methods over the past decade and their combination with established molecular analysis and structure determination techniques paves the way for understanding of life processes, and is on the brink of emerging applications as molecular diagnostic tests and targeted therapeutics.

\section{Which recent discovery might prove most valuable to the field of bioanalytical research or beyond?}

There are so many new developments in our discipline that it is difficult to choose one and only one. The selection that follows is based on my personal taste and experience, but it also allows me to tackle an interesting point, namely how sometimes evolutionary development is unpredictable. Inductively coupled plasma mass spectrometry started its development in the 1980s as a tool for panoramic elemental trace analysis, gradually replacing spark, arc and glow discharge mass spectrometry for multi-elemental analysis. The method diversified later as a hyphenated method, as a sensitive detector for chromatography and speciation analysis. Potential applications expanded to non-destructive analysis and imaging when combined with laser ablation and as a method for isotopic ratio analysis, replacing thermal ionization for most applications. All these are predictable evolutionary trends for a method as it develops to a mature state. More unpredictable was that its use expanded to the ultra-sensitive determination of large biomolecules as a technique called mass cytometry in the analysis of single cells and, with the addition of laser ablation, mass cytometry imaging. This particular method is based on antibody-labelling of specific proteins with a specific isotopically enriched rare earth atom or mixture. It enables the simultaneous quantitative measurement or imaging of different proteins or other biomolecules at the cellular or subcellular level with high spatial resolution and high sensitivity.

Proteins are important molecules that play key roles in all life processes. In a single cell, they are expressed from the genetic code through transcriptomics, and can serve as biomarkers which can be used to distinguish between healthy and diseased tissues, revealing information about the disease state. Relative quantification can be achieved by comparing the abundance of protein signals in different physiological states. In this way, by simultaneously identifying and measuring a large number of proteins, mass cytometry and imaging could eventually find a place in future health care as a clinical tool for personalized medicine.

Mass cytometry imaging is superior to its competitor, fluorescence microscopy, in which fluorescent dyes are tagged to the specific protein molecule. This is because the organic dyes face a 'color barrier': within the available spectral range, line broadening limits the number of molecules that can be simultaneously measured to a maximum of 4 or 5 . By contrast, the potential of mass cytometry is, in principle, quasi-unlimited. Disregarding conventional analytical characteristics such as sensitivity, specificity, speed, etc., for such multi-modal applications, mass cytometry is by far superior in performance to fluorescence cytometry.

When evaluating analytical methodologies, such as the ones above, for their fitness-of-purpose to solve particular problems in complex data intensive chemical analysis, it is important to have a specific descriptor in addition to conventional descriptive terms (such as sensitivity, specificity, precision or accuracy) whose significance is limited to a single measurement and a single analyte. As there is no descriptive term available right now, I introduce here by default a concept that I call commensurability. Perhaps learned societies such as IUPAC or EuChemS and their working parties could eventually agree on a more appropriate descriptor. The commensurability of an analytical method underlines the extent to which the collection of a number of analytical results have a given relationship to each other or to other measured entities (spatial information, structural or morphological information or physical data) and are able to help in solving a given scientific, societal or technological problem. For instance, mass cytometry and imaging can be considered more commensurate than fluorescence cytometry/imaging because it allows for the simultaneous measurement of hundreds of molecules instead of only a few and, hence, is superior for applications such as personalized medicine. The term commensurability as introduced here refers to the commensurability concept in mathematics or, in another context, in analytic philosophy, where it was introduced independently by Paul Feyerabend and Thomas Kuhn.

\section{In your opinion, does/did your generation have to respond to the publish or perish "pressure," and what is your advice for young analytical scientists today?}

I do not particularly like — who really does? - the term "publish or perish" with its harsh connotation of indiscriminate "struggle and survival of the fittest". However, ambition and competition are necessary ingredients for productivity and quality. One cannot pretend to need ca. $2 \%$ of the GDP without proper quality control on performance. Scientific careers 
should be evaluated with wisdom and consideration, openness, objectiveness and fairness. Bibliographic tools are useful objective and quantitative indicators, within a more holistic evaluation process, for funding, appointments, promotions, tenure, prizes and so on.

Scientific progress is driven by new scientific questions and these require fresh ways of thinking. Young analytical chemists should be alert for the big developments ahead of them. In the first place, there is the scale and complexity of many of the most interesting research problems. These move beyond the confines of a particular discipline. The focus should be on interdisciplinary teams rather than on individuals with an interdisciplinary education, thus reinforcing standard disciplinary boundaries, rather than breaking them down. Measurement science, which includes analytical chemistry, is a crucial participant, even an enabler of multidisciplinary research. Analytical scientists will increasingly work in large collaborations; they will need communication skills and collective self-scrutiny. Second, analytical chemistry is moving deeper into Pasteur's quadrant with work closer to potential applications. It plays a key role in research and innovation, and their transition into industry and post-industrial society. Chemical analysis will be a major contributor to addressing the huge problems facing the world today, which is increasingly out of equilibrium with its natural environment. The coming "decarbonization" of the global economy and the realization of sustainable development, assisting in the many societal changes needed to reduce our impacts on the earth, are the most important fields of work. Analytical chemistry is a major contributor in this transformation.

In summary, researchers of the coming generation should think out-of-the-box, in the conventional meaning of this concept - in the way they dare to think creatively — but also on how they should learn to think and act on cross-disciplinary and multi-disciplinary problems. Doing this, they should color inside the lines, think or act in accordance with the principles of their discipline and the basics of analytical chemistry as a measurement science.

\section{And last but not least, talking not only to the researcher: What do you enjoy most when you have time to spare?}

As a retired and aging scientist, I have a lot of spare time and few real obligations or deadlines. I now have plenty of time for history and the lessons we can draw from it. The future never copies the past, but at least, it sometimes rhymes with it. The visual arts, especially drawing and painting, have always been my interest. It is fortunate that there are almost limitless possibilities to view them in full detail in museums, but also, with the present-day technological opportunities, at home, from an easy chair. But I also keep following aspects of my discipline and developments of science and research in general. This because I like it; there is joy in research after retirement.

But digging deeper into the question, which hints at retirement, twelve years as the academic head of a university taught me how painfully divided on this matter the interests of the university and those of retiring professors can be. Getting out of a research career is a lot more difficult than stumbling into it when young. Perhaps, that too requires a source of inspiration. For this I hope to follow in the footsteps of Professor Wilhelm Fresenius (1913-2004), the great analytical chemist and former editor of this journal for so many years. He remained interested in science and followed the evolution of our discipline almost up to the end of his life at the age of 91. I had many opportunities to meet and have discussions with him in the late 1980s and the 1990s at conferences that he kept attending, from some distance as an interested but noninterfering observer, keeping himself away from the action. I hope to follow his example for the remaining time that is granted to me.

Freddy, thank you for sharing your thoughts with us! 